\title{
RhoV mediates apoptosis of RAW264.7 macrophages caused by osteoclast differentiation
}

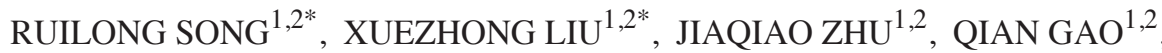 \\ QICHAO WANG ${ }^{1,2}$, JIAMING ZHANG ${ }^{1,2}$, DONG WANG ${ }^{1,2}$, LAIYANG CHENG ${ }^{1,2}$, \\ DI HU ${ }^{1,2}$, YAN YUAN ${ }^{1,2}$, JIANHONG GU ${ }^{1,2}$ and ZONGPING LIU ${ }^{1,2}$ \\ ${ }^{1}$ College of Veterinary Medicine, Yangzhou University; ${ }^{2}$ Jiangsu Co-innovation Center for Prevention and Control \\ of Important Animal Infectious Diseases and Zoonoses, Yangzhou, Jiangsu 225009, P.R. China
}

Received January 21, 2014; Accepted August 14, 2014

DOI: $10.3892 / \mathrm{mmr} .2014 .2817$

\begin{abstract}
Macrophages, a type of immune cell, are the precursors of osteoclasts, and have important roles in bone remodeling and the immune system. In the present study, the RAW264.7 cell line was used as a macrophage model in order to study the macrophage changes during osteoclastogenesis. Receptor activator of nuclear factor $\kappa \mathrm{B}$ ligand (RANKL) and macrophage colony-stimulating factor (M-CSF) induce the formation of osteoclasts from several precursor cells. Observation of RAW264.7 macrophage osteoclastogenesis under the induction of RANKL and M-CSF revealed that except the few RAW264.7 macrophages that were differentiated into osteoclasts, almost all undifferentiated RAW264.7 macrophages underwent apoptosis. BRL-3A cells have no differentiation ability, and RANKL and M-CSF treatments did not induce BRL-3A cell apoptosis. When osteoprotegerin (OPG) was used to completely inhibit the differentiation of RAW264.7 macrophages to osteoclasts, apoptosis did not occur amongst the RAW264.7 macrophages despite the action of RANKL and M-CSF. Rac1, RhoA and RhoV are apoptosis-associated genes in the Rho guanosine triphosphate (GTP)ase family. Their expression levels were detected using quantitative polymerase chain reaction (qPCR). During the process of osteoclast differentiation, the mRNA expression of RhoV was significantly upregulated, while apoptosis occurred in a large proportion of macrophages. However, when macrophage apoptosis was inhibited by OPG, RhoV expression was significantly downregulated. Conversely, Rac1 and RhoA expression did not vary in correspondence with the
\end{abstract}

Correspondence to: Professor Zongping Liu, College of Veterinary Medicine, Yangzhou University, 12 Wenhui East Road, Yangzhou, Jiangsu 225009, P.R. China

E-mail: liuzongping@yzu.edu.cn

${ }^{*}$ Contributed equally

Key words: osteoclast, apoptosis, differentiation, osteoprotegerin, RhoV apoptotic rate of the RAW264.7 macrophages. In conclusion, differentiation of RAW264.7 macrophages into osteoclasts resulted in their apoptosis. OPG inhibited RAW264.7 macrophage differentiation into osteoclasts, and thereby inhibited the apoptosis of RAW264.7 macrophages. RhoV mediated the apoptosis of RAW264.7 macrophages during osteoclast differentiation.

\section{Introduction}

Osteoclasts are cells of the macrophage lineage and are characterized by unique bone resorption activity (1-5). Since osteoclasts are terminal cells and possess no proliferation ability, they can only be derived from the differentiation of macrophages $(1,3)$. Previous studies indicated that in the presence of receptor activator of nuclear factor $\kappa \mathrm{B}$ ligand (RANKL) and macrophage colony-stimulating factor (M-CSF), osteoclasts are able to be formed by the differentiation of several monocyte/macrophage precursors $(1,6-8)$. Osteoclast differentiation and bone resorption is mainly regulated by osteoprotegerin (OPG)/RANKL/receptor activator of nuclear factor $\kappa \mathrm{B}$ (RANK) system (9-11), in which RANKL and OPG are secreted by osteoblasts $(4,12)$. RANKL binds to its receptor activator RANK in the osteoclast precursors, thereby inducing the differentiation of macrophages into osteoclasts. OPG is the decoy receptor of RANKL and competes with RANK to bind RANKL, with antagonist effects to RANK. It is through this mechanism that osteoclast differentiation is inhibited $(9,13)$. In addition, OPG promotes the apoptosis of osteoclasts by inhibiting the formation of the ruffled border in mature osteoclasts (14-16). However, the effects of OPG on osteoclast precursors in the process of osteoclast differentiation remain to be elucidated.

In relevant studies of osteoclasts, RAW264.7 is the most commonly used osteoclast precursor cell line $(12,17,18)$. Macrophages are the precursors of osteoclasts and also a type of immune cell. Macrophages have important roles in bone remodeling and the immune system $(3,19)$. Numerous studies have focused on elucidating the mechanisms underlying the differentiation of macrophages into osteoclasts in the presence of RANKL and M-CSF (6-10,20). However, the changes of the RAW264.7 macrophages that do not differentiate into 
osteoclasts have not been studied during macrophage-osteoclast differentiation, to the best of our knowledge.

Rho guanosine triphosphate (GTP)-ases are among the molecular switches involved in signal transduction by membrane receptors. Rho GTPases contain high-affinity sites that bind to GTP and guanosine diphosphate (GDP). They are activated by GTP binding and are deactivated by GDP binding. Rho GTPases act as molecular switches between the activated and the deactivated states. Rho GTPases are associated with the regulation of various processes in the organisms, including cell apoptosis $(21,22)$. The Rho GTPase family comprises 22 members (23), which are divided into a classical type and an atypical type. Rac1 and RhoA are classical Rho GTPases, while RhoV is an atypical Rho GTPase $(24,25)$. It has been reported that Rho GTPases, including Racl (26), RhoA (24.25) and RhoV (27), have important roles in cell apoptosis.

In the present study, the changes of RAW264.7 macrophages that did not differentiate into osteoclasts and the function of Rho GTPases in these changes were investigated.

\section{Materials and methods}

Morphological observations. RAW264.7 and BRL-3A cell lines were purchased from the Type Culture Collection of the Chinese Academy of Sciences (Shanghai, China).

In order to observe the morphological alterations of RAW264.7 macrophages during the differentiation into osteoclasts, the whole process of osteoclast differentiation was studied. RAW264.7 macrophages were seeded into six-well plates containing $\alpha$-minimum essential medium (MEM; Gibco Life Technologies, Carlsbad, CA, USA) supplemented with $50 \mathrm{ng} / \mathrm{ml}$ M-CSF (Peprotech, Inc., Rocky Hill, NJ, USA) and $60 \mathrm{ng} / \mathrm{ml}$ RANKL (Peprotech) at a cell density of 1562.5 cells $/ \mathrm{cm}^{2}$ (identical cell density was used in all experiments). The morphological changes of RAW264.7 were observed by a Hoffman microscope (Leica DMI3000; Leica Microsystems GmbH, Wetzlar, Germany) on days one to four.

To determine the cause of RAW264.7 macrophage apoptosis, the BRL-3A cell line, which has no differentiation capacity, was analyzed in order to distinguish between the potentially toxic activity of RANKL and the differentiation induced by RANKL. Subsequently, RAW264.7 and BRL-3A cells were plated with $\alpha$-MEM supplemented with $50 \mathrm{ng} / \mathrm{ml} \mathrm{M-CSF}, 60 \mathrm{ng} / \mathrm{ml} \mathrm{RANKL}$ and $50 \mathrm{ng} / \mathrm{ml} \mathrm{M-CSF}$ plus $60 \mathrm{ng} / \mathrm{ml}$ RANKL. No cytokines were added to cells in the control group. The cells were cultured for four days. The subsequent changes in the macrophages in each group were observed using a Hoffman microscope.

Immunofluorescent staining. In order to further study the association between differentiation and apoptosis, OPG was introduced to inhibit the differentiation of RAW264.7 cells. The RAW264.7 cells were cultured in $\alpha$-MEM supplemented with $50 \mathrm{ng} / \mathrm{ml} \mathrm{M-CSF}$ and $60 \mathrm{ng} / \mathrm{ml} \mathrm{RANKL}$ or $50 \mathrm{ng} / \mathrm{ml}$ M-CSF, $60 \mathrm{ng} / \mathrm{ml} \mathrm{RANKL}$ and $40 \mathrm{ng} / \mathrm{ml}$ OPG (Peprotech). The former treatment was the inducer treatment group and the latter was the OPG inhibition group. No cytokines were added to cells in the control group. BRL-3A cells were cultured in
$\alpha$-MEM supplemented with $50 \mathrm{ng} / \mathrm{ml} \mathrm{M-CSF}$ and $60 \mathrm{ng} / \mathrm{ml}$ RANKL. All cells were cultured for four days and were finally fixed in paraformaldehyde for $30 \mathrm{~min}$. Propidium iodide (PI; Sigma-Aldrich, St Louis, MO, USA) and Hoechst 33258 (Sigma-Aldrich) double staining was performed for $15 \mathrm{~min}$, respectively. Images were captured using a Leica DMI3000 inverted fluorescence microscope.

DNA Ladder experiment. In order to make the present study more rigorous, the DNA Ladder experiment was used to validate the association between differentiation and apoptosis. The RAW264.7 cells were cultured in $\alpha$-MEM supplemented with $50 \mathrm{ng} / \mathrm{ml} \mathrm{M-CSF}$ and $60 \mathrm{ng} / \mathrm{ml}$ RANKL or supplemented with $50 \mathrm{ng} / \mathrm{ml} \mathrm{M-CSF}, 60 \mathrm{ng} / \mathrm{ml}$ RANK and $40 \mathrm{ng} / \mathrm{ml}$ OPG. The former was the inducer treatment group, and the latter was the OPG inhibition group. No cytokines were added to cells in the control group. All cells were cultured for four days. DNA extraction was performed using a Wizard Genomic DNA Purification kit (Promega Corporation, Madison, WI, USA). The extracted DNA was separated by $2 \%$ agarose gel electrophoresis for $50 \mathrm{~min}$ at $90 \mathrm{~V}$. DNA bands were detected by a gel imaging system (Bio-Rad Laboratories, Inc., Hercules, CA, USA).

Fluorescence quantitative polymerase chain reaction ( $q P C R$ ) analysis. In order to study the roles of RhoA, Rac1 and RhoV in the process of RAW264.7 macrophage apoptosis caused by osteoclast differentiation, fluorescence qPCR was used to detect the mRNA expression levels of each.

The mRNA expression levels of RhoA, Racl and RhoV were detected during the process of osteoclast differentiation. The RAW264.7 macrophages were cultured by the method described for observing the morphological changes of macrophages during differentiation.mRNA extraction was performed on days 1-4 using TRIzol (Invitrogen Life Technologies, Carlsbad, CA, USA). Subsequently, reverse transcription was performed using the PrimeScript RT reagent kit with genomic DNA eraser (Takara Bio, Inc., Otsu, Japan). A real-time PCR system (Applied Biosystems 7500, Life Technologies, Foster City, CA, USA) was used to detect mRNA expression levels of Rac1, RhoA and RhoV. The primers were designed using Primer Premier 5 from published gene sequences (http://www. ncbi.nlm.nih.gov/) and shown in Table I.

The expression of mRNA of RhoA, Racl and RhoV in undifferentiated, differentiated and differentiation-inhibited RAW264.7 macrophages was detected. RAW264.7 cells were seeded into a six-well plate using the method described in the DNA Ladder experiment. The cells were cultured for four days, following which mRNA extraction and reverse transcription were performed. The mRNA expression levels of Rac1, RhoA and RhoV were detected using qPCR.

Statistical analysis. In the present study, each experiment was conducted in triplicate. The mRNA expression level results were analyzed by comparison of their $2^{-\Delta \Delta C t}$ values. Results are represented statistically as the mean \pm standard deviation. Significance was assessed using one-way analysis of variance (ANOVA). The results were compared between groups using ANOVA and Fisher's least significant difference post-hoc tests following appropriate transformation to normalized data and 
Table I. Primers used in quantitative polymerase chain reaction analyses.

\begin{tabular}{lcll}
\hline Gene & Accession no. & \multicolumn{1}{c}{ Upstream } & Downstream \\
\hline RhoA & NM_016802 & CAAGGACCAGTTCCCAGAGG & CGCAGGCGGTCATAATCTTC \\
Rac1 & NM_009007 & GCCTGCTCATCAGTTACACG & GACGCAATCTGTCATAATCTTC \\
RhoV & NM_145530 & GCAGCCTCATCGTCAGCTACAC & GAAGCAAGCCAGAAAGACATCG \\
GAPDH & GU214026 & ATGGTGAAGGTCGGTGTG & TGAAGGGGTCGTTGATGG
\end{tabular}

A:

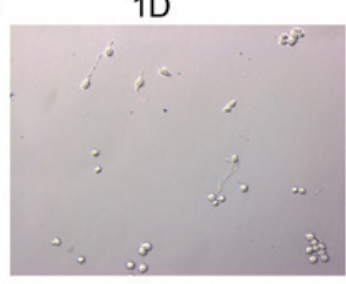

$1 \mathrm{D}$

B

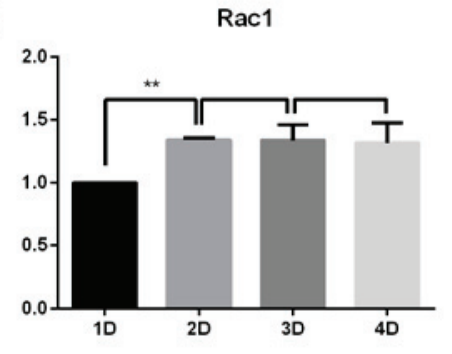

2D

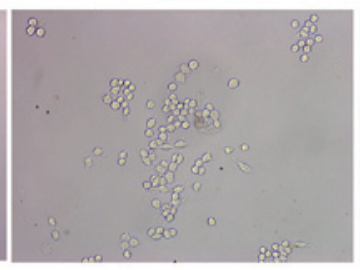

3D

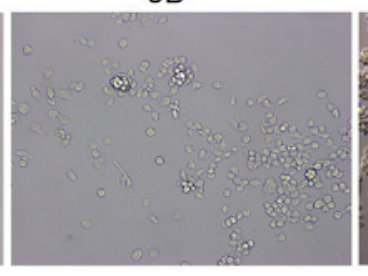

4D

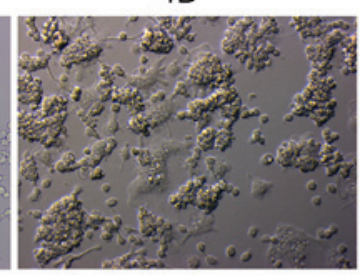

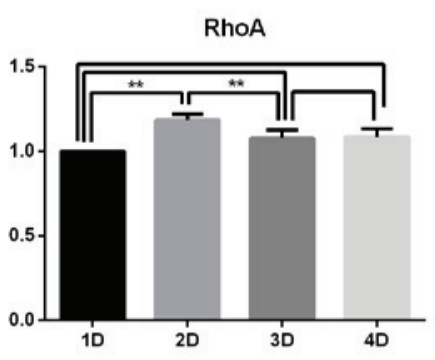

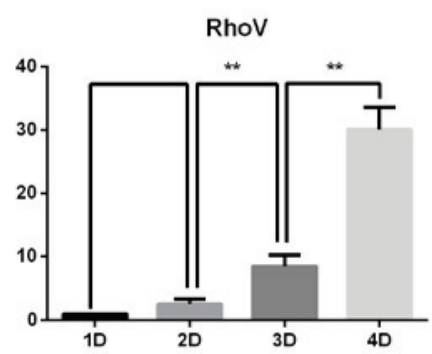

Figure 1. Morphological changes and the mRNA expression levels of Rac1, RhoA and RhoV during osteoclast differentiation. (A) Morphological changes of the cells were observed with a microscope on days 1-4 of the culture (magnification x200). (B) mRNA extraction was performed on days 1-4 and mRNA expression levels of Rac1, RhoA and RhoV were detected. Pairs of the identical lower- and upper-case letters indicate an insignificant difference between the groups. ${ }^{*} \mathrm{P}<0.05 ;{ }^{* *} \mathrm{P}<0.01$.

equalized variance where necessary. Statistical analysis was performed using Statistical Analysis System (SAS) 9.1.3 (SAS Institute, Inc., Cary, NC, USA); $\mathrm{P}<0.05$ and $\mathrm{P}<0.01$ were considered to indicate a statistically significant difference between values. In the qPCR experiments for osteoclast differentiation, comparisons were made among the expression levels of Racl, RhoA and RhoV at different stages of differentiation. In the qPCR experiment observing the effects of OPG on undifferentiated RAW264.7, comparisons were made between the expression of Racl, RhoA and RhoV within the inducer group and the control group, along with that between the inducer treatment group and OPG inhibition group.

\section{Results}

Process of osteoclast differentiation. The various changes of the cells in the induction group were observed on days 1-4 using a Hoffman microscope. As indicated in Fig. 1A, the number of RAW264.7 macrophages increased with the extension of induction time. No osteoclasts were produced in the first three days. On day four, a large number of osteoclasts had developed. Simultaneously, there was a notable level of apoptosis of RAW264.7 cells.
In order to investigate the roles of Rac1, RhoA and RhoV in the apoptosis of RAW264.7 cells that did not differentiate into osteoclasts, the mRNA expression of Rac1, RhoA and RhoV was detected by qPCR. As indicated in Fig. 1B, the mRNA expression of Racl and RhoA was maintained at a constant level following day two, where a slight upregulation was observed. The mRNA expression of RhoV was continually upregulated with the extension of the induction treatment. The mRNA expression of RhoV on day four was almost 30 times that of the expression levels on day one.

Effects of M-CSF, RANKL and the combination of the two inducers on cell differentiation and apoptosis. The effects of M-CSF and RANKL alone and the combined use of the two inducers on the cell differentiation and apoptosis were investigated by observing changes in the cells of each group following four days of culture using a Hoffman microscope. The BRL-3A liver cells were used as the control to discriminate between the apoptosis-inducing effect of the inducers and the apoptosis caused by the differentiation of RAW264.7 cells.

As indicated in Fig. 2, following four-day culture in $\alpha$-MEM supplemented with RANKL or supplemented with RANKL and M-CSF, RAW264.7 cells in the two groups demonstrated a scattered distribution. A large amount of 


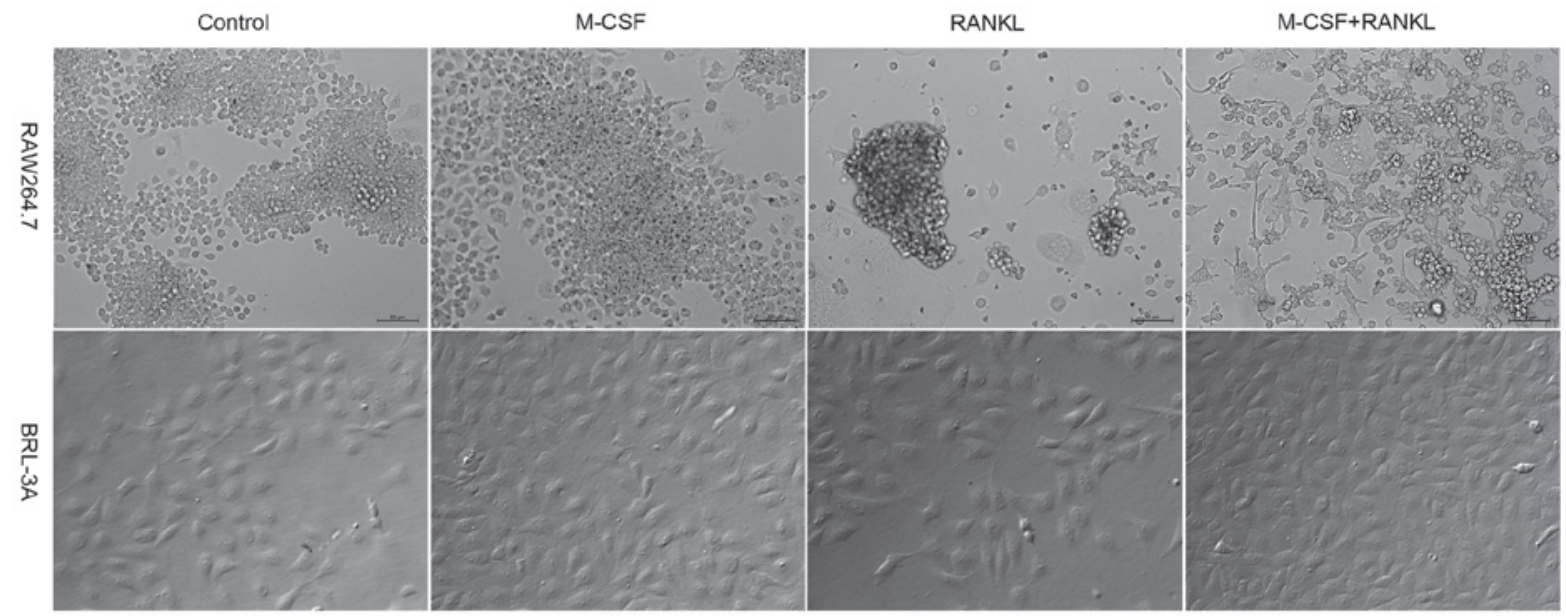

Figure 2. Effects of M-CSF, RANKL and the combination of the two inducers on the apoptotic rate of RAW264.7 and BRL-3A cells. The cells were photographed using an inverted microscope following four days of culture (magnification x200). M-CSF, macrophage colony-stimulating factor; RANKL, receptor activator of nuclear factor $\kappa \mathrm{B}$ ligand.

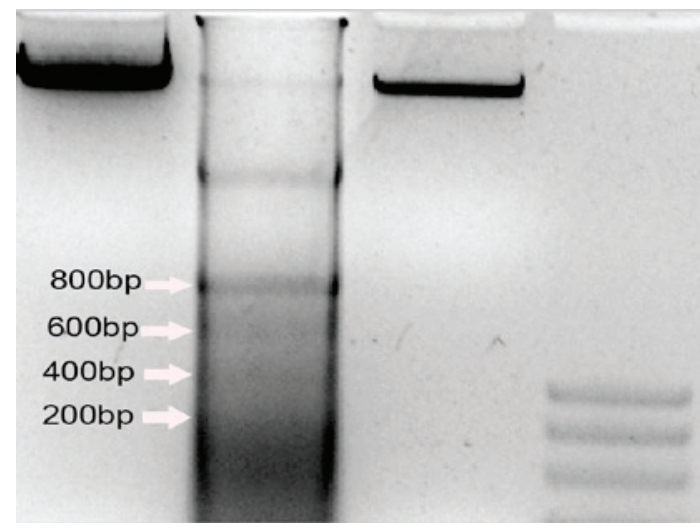

Figure 3. Agarose gel electrophoresis of DNA fragmentation. Lanes: 1, control; 2, M-CSF+RANKL; 3, M-CSF+RANKL+osteoprotegerin; M, marker. M-CSF, macrophage colony-stimulating factor; RANKL, receptor activator of nuclear factor $\mathrm{\kappa B}$ ligand.

osteoclasts were produced; however, a significant percentage of RAW264.7 macrophages demonstrated apoptosis indicated by detached or floating cells. This result was consistent with that observed on day four in Fig. 1A. In the control group and the M-CSF treatment group, the cells grew in aggregates, no osteoclasts were produced and no apoptosis of RAW264.7 cells was observed. BRL-3A cells have no differentiation ability. In all four groups of BRL-3A cells, cell growth was normal and no apoptosis was observed as in the case of RAW264.7. It was also found that the cell count in the four groups receiving M-CSF treatment was higher than that in the groups without M-CSF treatment. This is because M-CSF promotes the division and proliferation of macrophages.

Detection of cell apoptosis in the control, inducer treatment and OPG inhibition groups by DNA Ladder. The 200 bp DNA ladder is a marker that differentiates cell necrosis from cell apoptosis. DNA fragmentation was detected in the control, M-CSF+RANKL and OPG+M-CSF+RANKL groups to reveal the effects of osteoclast differentiation on the apoptosis of RAW264.7 macrophages. The DNA Ladder was produced in the M-CSF+RANKL group, but not in the $\mathrm{OPG}+\mathrm{M}-\mathrm{CSF}+\mathrm{RANKL}$ or control groups (Fig. 3).

Detection of RAW264.7 cell apoptosis by Hoechst 33258 and PI double staining. PI and Hoechst 33258 bind to nuclear DNA. PI is unable to pass through the cell membrane of live cells, while Hoechst 33258 is a membrane-permeable fluorescent dye. For those cells in necrosis or late-stage apoptosis, the cell membrane is damaged and therefore, the respective cell is stained red by PI. The effects of the differentiation of RAW264.7 macrophages on their apoptotic rate was further studied by PI and Hoechst 33258 double staining.

As exhibited in Fig. 4, the BRL-3A cells were not stained by $\mathrm{PI}$ in the M-CSF+RANKL treatment group, which indicated that no apoptosis of BRL-3A cells occurred in the presence of the inducers. This result was consistent with that exhibited in Fig. 2. Following M-CSF and RANKL treatments alone, a large number of RAW264.7 macrophages were stained red by PI. This indicated that marked apoptosis had occurred in RAW264.7 cells following the induction. Conversely, the cells in the control group and the OPG inhibition group were not stained by PI. This indicated that no apoptosis of the cells occurred in these groups, consistent with the results of the DNA Ladder detection experiment (Fig. 3).

mRNA expression levels of Racl, RhoA and RhoV in the control, inducer treatment and OPG inhibition groups. In order to investigate the roles of Rac1, RhoA and RhoV in the process of RAW264.7 cell apoptosis caused by RAW264.7 differentiation, mRNA expression of Rac1, RhoA and RhoV was detected using qPCR in the control, M-CSF+RANKL treatment and $\mathrm{OPG}+\mathrm{M}-\mathrm{CSF}+\mathrm{RANKL}$ groups (Fig. 5).

It was demonstrated that the mRNA expression of Rac1 and RhoA exhibited significant decreases in the inducer treatment and OPG inhibition groups. Conversely, RhoV mRNA expression was significantly upregulated in the inducer treatment group. Compared with the inducer treatment group, RhoV mRNA expression was significantly downregulated in the OPG inhibition group. 


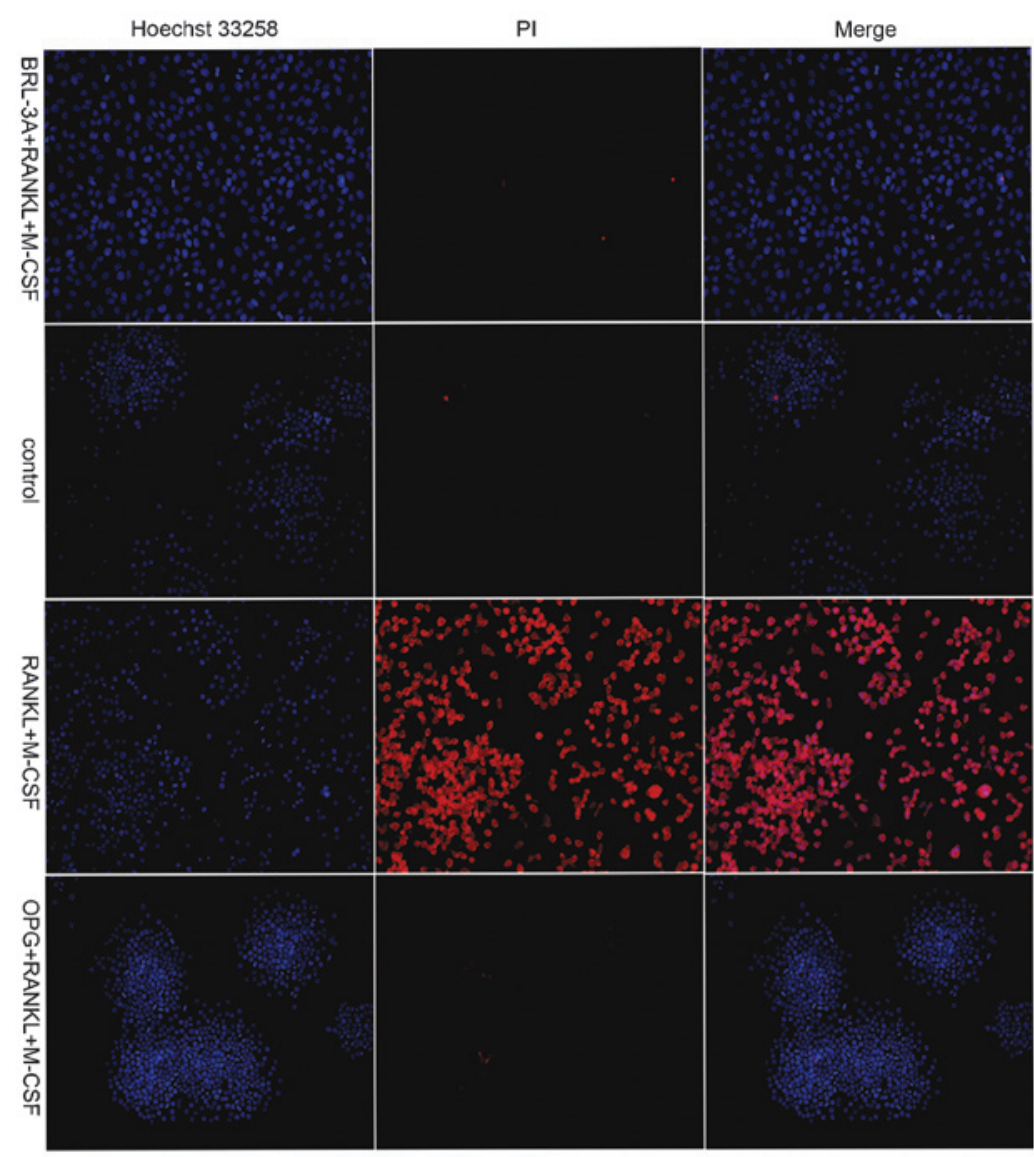

Figure 4. Apoptosis of RAW264.7 macrophages caused by osteoclast differentiation. The association between the differentiation and apoptosis of RAW264.7 cells was detected by Hoechst 33258 (blue) and PI (red) double staining (magnification, x200). Top row, BRL-3A cells; subsequent three rows, RAW264.7 cells. Images were captured using an inverted fluorescence microscope. PI, propidium iodide; M-CSF, macrophage colony-stimulating factor; RANKL, receptor activator of nuclear factor $\mathrm{\kappa B}$ ligand; OPG, osteoprotegerin.
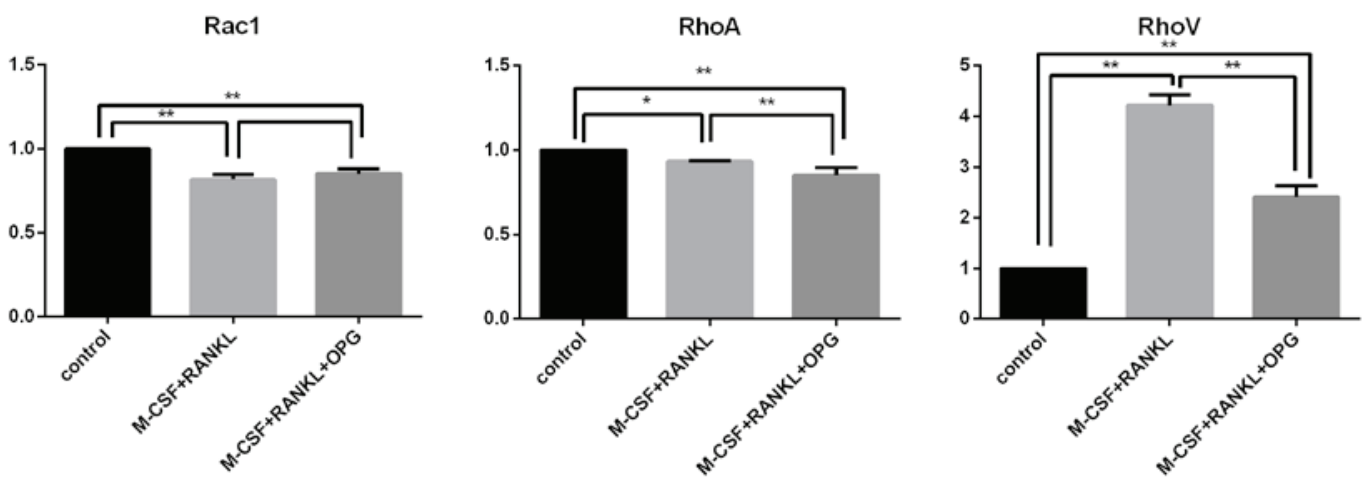

Figure 5. mRNA expression of Rac1, RhoA and RhoV in undifferentiated, differentiated and differentiation-inhibited RAW264.7 macrophages. mRNA expression of Rac1, RhoA and RhoV was detected following four-day culture of RAW264.7 with different cytokines as indicated. Identical pairs of lower- and upper-case letters indicate an insignificant difference between the groups. ${ }^{*} \mathrm{P}<0.05 ;{ }^{* *} \mathrm{P}<0.01$. M-CSF, macrophage colony-stimulating factor; RANKL, receptor activator of nuclear factor $\mathrm{\kappa B}$ ligand; $\mathrm{OPG}$, osteoprotegerin.

\section{Discussion}

In the present study, the effects of osteoclast differentiation on RAW264.7 macrophages were investigated. With the exception of a number of RAW264.7 macrophages which differentiated into osteoclasts following RANKL and M-CSF induction, nearly all RAW264.7 macrophages underwent apoptosis. It was also observed that when OPG was used to inhibit the differentiation of RAW264.7 macrophages, the apoptosis of RAW264.7 macrophages was inhibited. Furthermore, RhoV was the mediator of the apoptosis of RAW264.7 macrophages caused by their differentiation. 
In bones, RANKL and M-CSF are produced by cells of osteoblastic lineage. RANKL and M-CSF are able to activate a series of complex reaction of macrophages and induce their differentiation into osteoclasts. The oversecretion of RANKL and M-CSF leads to the abnormal proliferation of osteoclasts and hence a variety of bone diseases, including rheumatoid arthritis, osteoporosis and multiple myeloma $(2,28)$. There is a reduction in estrogen levels in females in the menopausal period (29), which leads to an increase in RANKL expression. Therefore, this results in excessive differentiation of macrophages into osteoclasts $(30,31)$. According to existing studies, the probability of occurrence of various diseases was increased during the menopausal period due to decreased immunity (32-35). Unexpectedly, a large amount of osteoclasts were produced on day four, under the induction of RANKL and M-CSF. Simultaneously, a considerable amount of apoptosis of RAW264.7 macrophages occurred. Subsequently, the effects exposure to of M-CSF and/or RANKL on the apoptosis of RAW264.7 macrophages were studied. It was found that the undifferentiated RAW264.7 macrophages underwent apoptosis in the two groups receiving RANKL treatment. This process was accompanied by the production of large quantities of osteoclasts. In order elucidate whether the cause of the apoptosis of RAW264.7 macrophages was RANKL induction or differentiation of RAW264.7, the BRL-3A cell line, which has no differentiation capability, was used as a control group. The results indicated that neither RANKL nor M-CSF induced the apoptosis of BRL-3A cells. Furthermore, RANKL itself is a substance produced in normal organisms. These combined results indicated that the differentiation of RAW264.7 macrophages induced the observed apoptosis. Whether the high levels of apoptosis of macrophages are the direct reason for the low immunity associated with the menopausal period remains to be elucidated by further experiments.

OPG is also produced by cells of osteoblastic lineage, and is able to inhibit the differentiation of macrophages into osteoclasts $(9,11)$. To verify the hypothesis that the differentiation of RAW264.7 macrophages caused their apoptosis, OPG was added into the RANKL+M-CSF induction treatment group in order to inhibit the differentiation of RAW264.7 macrophages. PI and Hoechst 33258 double staining and DNA Ladder experiments were performed. In the inducer treatment group, a large amount of undifferentiated RAW264.7 macrophages experienced apoptosis. However, in the OPG inhibition group and the control group without any cytokines, no apoptosis of RAW264.7 macrophages was observed. These results indicated that the differentiation of RAW264.7 macrophages into osteoclasts itself induced apoptosis. By inhibiting the differentiation of RAW264.7 macrophages, OPG inhibited the differentiation-dependent RAW264.7 apoptosis.

Rho GTPases regulate diverse metabolic processes in cells, including dynamic changes of the cytoskeleton, cell adhesion, gene expression and cell apoptosis $(21,26,36,37)$. Rho GTPases, including RhoA, Racl and RhoV, have important roles in cell apoptosis (24-27). The mRNA expression levels of RhoA, Racl and RhoV during the process of osteoclast differentiation were detected in order to investigate the effects of the inducers RANKL and M-CSF, and the inhibitor OPG on the apoptotic rate of RAW264.7 macrophages. Of note, the apoptosis of undifferentiated RAW264.7 macrophages occurred on day four of the culture. Compared with day one, the mRNA expression of RhoA and Racl on day four demonstrated only minor upregulation and subsequently remained at this level. In the qPCR experiment examining the effects of OPG on undifferentiated RAW264.7, the mRNA expression levels of RhoA and Racl showed significant downregulation in the inducer treatment groups and OPG inhibition group compared with those in the control group. This change was inverse to the apoptotic rate of the cells. It was demonstrated that the apoptotic rate of RAW264.7 macrophages induced by their differentiation did not necessarily involve the action of RhoA and Rac1. However, on day four, when high levels of apoptosis of RAW264.7 cells were present, the mRNA expression of RhoV was significantly upregulated. In the Hoechst 33258 and PI double staining experiment, and the DNA Ladder experiment, compared with the control group and OPG inhibition group where no apoptosis occurred, the cells in the inducer treatment group experienced notable apoptosis. The mRNA expression of RhoV was also significantly upregulated, corresponding to the apoptotic rate of the cells. These results demonstrated that $\mathrm{RhoV}$ had an important role in regulating the apoptosis of RAW264.7 macrophages caused by their differentiation.

In the present study, the apoptotic rate of RAW264.7 macrophages in the presence of RANKL was investigated. RANKL induced the differentiation of RAW264.7 macrophages into osteoclasts. In conclusion, it was demonstrated that the differentiation of RAW264.7 macrophages itself was the cause of the high levels of apoptosis. The addition of OPG inhibited the differentiation of RAW264.7 macrophages into osteoclasts, which thereby inhibited the apoptosis of RAW264.7 macrophages. Additionally, in the process of osteoclast differentiation, RhoV mediated the RAW264.7 macrophage apoptosis.

\section{Acknowledgements}

The present study was supported by the National Natural Science Foundation of China (nos. 31172373, 31302154 and 31372495), the Specialized Research Fund for the Doctoral Program of Higher Education (no. 20113250110003) and a project funded by the Priority Academic Program Development of Jiangsu Higher Education Institutions and the Graduate Innovation Project of Jiangsu Province (no. CXZZ12_0917).

\section{References}

1. Husheem M, Nyman JK, Vääräniemi J, Vaananen HK and Hentunen TA: Characterization of circulating human osteoclast progenitors: development of in vitro resorption assay. Calcif Tissue Int 76: 222-230, 2005.

2. Boyle WJ, Simonet WS and Lacey DL: Osteoclast differentiation and activation. Nature 423: 337-342, 2003.

3. Väänänen HK and Laitala-Leinonen T: Osteoclast lineage and function. Arch Biochem Biophys 473: 132-138, 2008.

4. Teitelbaum SL: Bone resorption by osteoclasts. Science 289: 1504-1508, 2000.

5. Väänänen HK, Zhao H, Mulari M and Halleen JM: The cell biology of osteoclast function. J Cell Sci 113: 377-381, 2000.

6. Jansen ID, Vermeer JA, Bloemen V, Stap J and Everts V: Osteoclast fusion and fission. Calcif Tissue Int 90: 515-522, 2012.

7. Yasuda H, Shima N, Nakagawa N, et al: Osteoclast differentiation factor is a ligand for osteoprotegerin/osteoclastogenesis-inhibitory factor and is identical to TRANCE/RANKL. Proc Natl Acad Sci USA 95: 3597-3602, 1998. 
8. Lacey DL, Timms E, Tan HL, et al: Osteoprotegerin ligand is a cytokine that regulates osteoclast differentiation and activation. Cell 93: 165-176, 1998.

9. Hofbauer LC, Kühne CA and Viereck V: The OPG/RANKL/RANK system in metabolic bone diseases. J Musculoskelet Neuronal Interact 4: 268-275, 2004.

10. Khosla S: Minireview: the OPG/RANKL/RANK system Endocrinology 142: 5050-5055, 2001.

11. Song R, Gu J, Liu X, et al: Inhibition of osteoclast bone resorption activity through osteoprotegerin-induced damage of the sealing zone. Int J Mol Med 34: 856-862, 2014

12. Singh PP, van der Kraan AG, Xu J, Gillespie MT and Quinn JM: Membrane-bound receptor activator of NFKB ligand (RANKL) activity displayed by osteoblasts is differentially regulated by osteolytic factors. Biochem Biophys Res Commun 422: 48-53, 2012.

13. Wang J, Chen TY, Qin S, Duan Y and Wang G: Inhibitory effect of metformin on bone metastasis of cancer via OPG/RANKL/RANK system. Med Hypotheses 81: 805-806, 2013.

14. Shiotani A, Takami M, Itoh K, Shibasaki Y and Sasaki T: Regulation of osteoclast differentiation and function by receptor activator of NFkB ligand and osteoprotegerin. Anat Rec 268 137-146, 2002.

15. Liu Z, Xu J, E L and Wang D: Ultrasound enhances the healing of orthodontically induced root resorption in rats. Angle Orthod 82: 48-55, 2012

16. Fu YX, Gu JH, Zhang YR, Tong XS, Zhao HY, Yuan Y, Liu XZ, Bian JC and Liu ZP: Influence of osteoprotegerin on differentiation, activation, and apoptosis of Gaoyou duck embryo osteoclasts in vitro. Poult Sci 92: 1613-1620, 2013.

17. Li CH, Zhao JX, Sun L, Yao ZQ, Deng XL, Liu R and Liu XY: AG490 inhibits NFATc1 expression and STAT3 activation during RANKL induced osteoclastogenesis. Biochem Biophys Res Commun 435: 533-539, 2013.

18. Robertson Remen KM, Gustafsson JA and Andersson G: The liver $\mathrm{X}$ receptor promotes macrophage differentiation and suppresses osteoclast formation in mouse RAW264.7 promyelocytic leukemia cells exposed to bacterial lipopolysaccharide. Biochem Biophys Res Commun 430: 375-380, 2013.

19. Sinningen K, Rauner M, Goettsch C, Al-Fakhri N, Schoppet M and Hofbauer LC: Monocytic expression of osteoclast-associated receptor (OSCAR) is induced in atherosclerotic mice and regulated by oxidized low-density lipoprotein in vitro. Biochem Biophys Res Commun 437: 314-318, 2013

20. Song RL, Liu XZ, Zhu JQ, et al: New roles of filopodia and podosomes in the differentiation and fusion process of osteoclasts. Genet Mol Res 13: 4776-4787, 2014.

21. Etienne-Manneville S and Hall A: Rho GTPases in cell biology. Nature 420: 629-635, 2002
22. Coxon FP and Rogers MJ: The role of prenylated small GTP-binding proteins in the regulation of osteoclast function. Calcif Tissue Int 72: 80-84, 2003.

23. Vega FM and Ridley AJ: SnapShot: Rho family GTPases. Cell 129: 1430, 2007.

24. Nakamura H, Hirata A, Tsuji T and Yamamoto T: Role of osteoclast extracellular signal-regulated kinase (ERK) in cell survival and maintenance of cell polarity. J Bone Miner Res 18: 1198-1205, 2003

25. Wang N, Robaye B, Agrawal A, Skerry TM, Boeynaems JM and Gartland A: Reduced bone turnover in mice lacking the P2Y(13) receptor of ADP. Mol Endocrinol 26: 142-152, 2012.

26. Fukuda A, Hikita A, Wakeyama H, Akiyama T, Oda $\mathrm{H}$, Nakamura K and Tanaka S: Regulation of osteoclast apoptosis and motility by small GTPase binding protein Rac1. J Bone Miner Res 20: 2245-2253, 2005.

27. Shepelev MV, Chernoff J and Korobko IV: Rho family GTPase $\mathrm{Chp} / \mathrm{RhoV}$ induces PC12 apoptotic cell death via JNK activation. Small GTPases 2: 17-26, 2011.

28. Chamoux E, Houde N, L'Eriger K and Roux S: Osteoprotegerin decreases human osteoclast apoptosis by inhibiting the TRAIL pathway. J Cell Physiol 216: 536-542, 2008.

29. Schindler AE: Climacteric symptoms and hormones. Gynecol Endocrinol 22: 151-154, 2006.

30. Blair HC, Robinson LJ and Zaidi M: Osteoclast signalling pathways. Biochem Biophys Res Commun 328: 728-738, 2005.

31. Syed F and Khosla S: Mechanisms of sex steroid effects on bone. Biochem Biophys Res Commun 328: 688-696, 2005.

32. Stolberg M: From the 'climacteric disease' to the 'male climacteric' The historical origins of a modern concept. Maturitas 58: 111-116, 2007.

33. Rollenhagen $\mathrm{C}$ and Asin SN: Enhanced HIV-1 replication in ex vivo ectocervical tissues from post-menopausal women correlates with increased inflammatory responses. Mucosal Immunol 4: 671-681, 2011.

34. Breuil V, Ticchioni M, Testa $\mathrm{J}$, et al: Immune changes in post-menopausal osteoporosis: the Immunos study. Osteoporos Int 21: 805-814, 2010.

35. Ambrogini E, Toraldo G and Marcocci C: Post-menopausal osteoporosis: is it an autoimmune disease? J Endocrinol Invest 28: 43-47, 2005.

36. Li F, Jiang Q, Shi KJ, Luo H, Yang Y and Xu CM: RhoA modulates functional and physical interaction between ROCK1 and Erk1/2 in selenite-induced apoptosis of leukaemia cells. Cell Death Dis 4: e708, 2013.

37. Ullah I, Lee HY, Kim MJ, Shah SA, Badshah H, Kim TH, Chung HJ, Yang BC and Kim MO: Rho GTPase activating protein 15 (arhGAP15) siRNA effect apoptosis-induced by ethanol in bovine fibroblast cells. Pak J Pharm Sci 26: 605-610, 2013. 